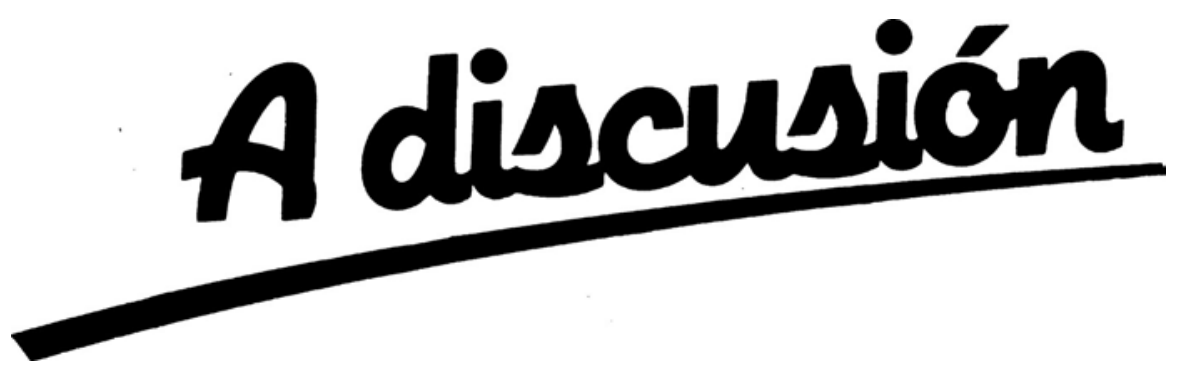

\title{
QUASI-GEOMETRIC DISCOUNTING: A CLOSED-FORM SOLUTION UNDER THE EXPONENTIAL UTILITY FUNCTION*
}

\section{Lilia Maliar and Serguei Maliar**}

WP-AD 2003-16

Correspondence to: Universidad de Alicante, Departamento de Fundamentos del Análisis Económico, Campus San Vicente del Raspeig, Ap. Correos, 99, 03080 Alicante (Spain). Emails: maliarl@merlin.fae.ua.es, maliars@merlin.fae.ua.es.

Editor: Instituto Valenciano de Investigaciones Económicas, S.A.

Primera Edición Abril 2003

Depósito Legal: V-1907-2003

IVIE working papers offer in advance the results of economic research under way in order to encourage a discussion process before sending them to scientific journals for their final publication.

* We have benefited from the comments of an anonymous referee and managing editor, Gianni De Fraja. All errors are ours. This research was partially supported by the Instituto Valenciano de Investigaciones Económicas and the Ministerio de Ciencia y Tecnología de España, BEC 2001-0535.

** Universidad de Alicante. 


\title{
QUASI-GEOMETRIC DISCOUNTING: A GLOSED-FORM SOLUTION UNDER THE EXPONENTIAL UTILITY FUNCTION
}

\author{
Lilia Maliar and Serguei Maliar
}

\begin{abstract}
This paper studies a discrete-time utility maximization problem of an infinitely-lived quasi-geometric consumer whose labor income is subject to uninsurable idiosyncratic productivity shocks. We restrict attention to a firstorder Markov recursive solution. We show that under the assumption of the exponential utility function, the problem of the quasi-geometric consumer admits a closed-form solution.
\end{abstract}

Keywords: quasi-geometric (quasi-hyperbolic) discounting, idiosyncratic shocks, closed-form solution.

JEL Classification: D91, E21, G11 
A large body of recent literature investigates the consumption-savings behavior of agents under the assumption of quasi-geometric (quasi-hyperbolic) discounting, e.g., Laibson (1997), Barro (1999), Harris and Laibson (2001), Krusell, Kuruş̧̧u and Smith (2002), Krusell and Smith (2003), Luttmer and Mariotti (2003). Krusell and Smith (2003) incorporate quasi-geometric discounting into a deterministic version of the standard infinite-horizon neoclassical growth model. In particular, they show that under the assumptions of logarithmic utility function, Cobb-Douglas production function and full depreciation of capital, the model allows for a closed-form solution.

This paper describes another model with quasi-geometric discounting that can be solved analytically. We study a discrete-time utility maximization problem of an infinitely-lived quasi-geometric consumer whose labor income is subject to uninsurable idiosyncratic productivity shocks. We restrict attention to a first-order Markov recursive solution. We show that under the assumption of the exponential utility function, the problem of such a consumer admits a closed-form solution. Our results can be viewed as an extension of the work of Caballero (1990), who derived a closed-form solution for the standard geometric-discounting case. 
At each date $t \in\{0,1,2, \ldots\}$, an agent solves the following problem

$$
\max _{\left\{c_{\tau}, a_{\tau+1}\right\}_{\tau=t}^{\infty}}\left\{u\left(c_{t}\right)+E_{t} \sum_{\tau=t}^{\infty} \beta \delta^{\tau+1-t} u\left(c_{\tau+1}\right)\right\}
$$

subject to

$$
c_{\tau}+a_{\tau+1}=w s_{\tau}+(1+r) a_{\tau}
$$

where initial condition $\left(a_{t}, s_{t}\right)$ is given. Here, $\beta>0$ and $\delta \in(0,1)$ are the discounting parameters; $c_{\tau}$ and $a_{\tau}$ are consumption and asset holdings, respectively; $s_{\tau}$ is an idiosyncratic productivity shock following a first-order Markov process; $r$ and $w$ are the interest rate and wage per unit of efficiency labor, respectively; $E_{\tau}$ is the expectation, conditional on all information about the agent's idiosyncratic shocks available at $\tau$.

It is typically assumed in the literature on quasi-geometric discounting that a discount factor, applied between today and tomorrow, is lower than the one, used on all dates advanced further in the future, $\beta<1$. This leads to the following form of time-inconsistency in preferences: the agent systematically plans to be patient (to save much) tomorrow, but as tomorrow arrives, she always changes her mind and behaves impatiently (saves little). One can also consider the opposite case, $\beta>1$, when the agent always behaves more patiently than she originally planned. The parameterization 
$\beta=1$ corresponds to the standard geometric-discounting case, when the agent is equally patient in both the short-run and the long-run.

We consider a recursive Markov solution to the problem (1), (2), such that in all periods, the agent decides on consumption according to the same decision rule $c_{t}=C\left(a_{t}, s_{t}\right)$. Then, without time subscripts, the recursive formulation of the individual problem is as follows:

$$
W(a, s)=\max _{c}\left\{u(c)+\beta \delta E\left[V\left(a^{\prime}, s^{\prime}\right) \mid s\right]\right\},
$$

where given $a, s$, the value function $V$ solves the functional equation

$$
V(a, s)=u[C(a, s)]+\delta E\left\{V\left[w s+(1+r) a-C(a, s) ; s^{\prime}\right] \mid s\right\}
$$

subject to the budget constraint

$$
a^{\prime}=w s+(1+r) a-c
$$

The problem (3) - (5) is to be solved for the unknown value functions $W(a, s), V(a, s)$ and the decision rule $C(a, s)$.

We shall assume that the agent has the exponential momentary utility function

$$
u\left(c_{t}\right)=-\frac{1}{\theta} \exp \left(-\theta c_{t}\right), \quad \theta>0
$$


As shown in Caballero (1990), under the assumption of standard geometric discounting $(\beta=1)$ and with some additional restrictions on the process for labor income shocks, the utility parametrization (6) leads to a closedform solution. In particular, a closed-form solution exists under a first-order autoregressive process

$$
s_{t+1}=\rho s_{t}+\varepsilon_{t+1}, \quad \text { with } \rho \in[0,1] \text { and } \varepsilon_{t+1} \sim N\left(0, \sigma^{2}\right) .
$$

With the following proposition, we establish the existence of a closed-form solution under the assumption of quasi-geometric discounting.

Proposition 1 Under (6), (7), the value functions $V$ and $W$ that solve the problem $(3)-(5)$ are given by

$$
V(a, s)=-\frac{1+\beta r}{\theta \beta r} \cdot \exp (-\theta c), \quad W(a, s)=-\frac{1+r}{\theta r} \cdot \exp (-\theta c),
$$

where $c=C(a, s)$ is given by

$$
c=r \cdot a+\frac{r w}{1+r-\rho} \cdot s-\frac{1}{\theta r} \ln [\delta(1+\beta r)]-\frac{\theta r w^{2} \sigma^{2}}{2(1+r-\rho)^{2}} .
$$

Proof. See Appendix. $\|$ 
The consumption function (9) and budget constraint (5) yield the following decision rule for asset holdings:

$$
a^{\prime}=a+\frac{(1-\rho) w}{1+r-\rho} \cdot s+\frac{1}{\theta r} \ln [\delta(1+\beta r)]+\frac{\theta r w^{2} \sigma^{2}}{2(1+r-\rho)^{2}}
$$

Equation (10) implies that individual asset holdings follow a random walk.

In our example, the properties of the optimal value functions $V, W$ and the decision rules $C, A$ are similar to those of the corresponding functions in the standard geometric discounting case. Specifically, all of the functions $V$, $W, C$ and $A$ are continuously differentiable, strictly increasing and concave ( $C$ and $A$ are not strictly concave, however). We shall also notice that our solution is interior, i.e., it satisfies the corresponding Euler equation.

The features of the solution (9) and (10) are as follows: For a given interest rate, the effect of the assumption of quasi-geometric discounting on the optimal allocations is reflected in the value of the term $\frac{1}{\theta r} \ln [\delta(1+\beta r)]$. Since this term increases in $\beta$, a larger value of the parameter $\beta$ implies a higher amount of savings. Therefore, among two agents, who are identical in all respects, except for the discounting parameter $\beta$, the agent with a larger $\beta$ will always choose to hold more assets than the one with a smaller $\beta$. Further, the role of the discounting parameters $\delta$ and $\beta$ in the individual consumption-savings behavior is indistinguishable: the decisions of 
a quasi-geometric consumer with the parameters $\delta$ and $\beta \neq 1$ are identical to those of a standard geometric consumer, $\widetilde{\beta}=1$, with the parameter $\widetilde{\delta}=\frac{\delta(1+\beta r)}{1+r}$. Finally, the assumption of quasi-geometric discounting does not affect the amount of savings for precautionary motives, which are defined as the difference between the agent's asset holdings with and without uncertainty. Indeed, according to (10), precautionary savings are given by the term $\frac{\theta r w^{2} \sigma^{2}}{2(1+r-\rho)^{2}}$, which is independent of the discounting parameter $\beta$.

\section{References}

[1] Barro, R., 1999, Ramsey meets Laibson in the neoclassical growth model, Quarterly Journal of Economics 114 (4), 1125-1152.

[2] Caballero, R., 1990, Consumption puzzles and precautionary savings, Journal of Monetary Economics 25, 113-136.

[3] Harris, C. and D. Laibson, 2001, Dynamic choices of hyperbolic consumers, Econometrica 69, 935-959.

[4] Krusell, P. and A. Smith, 2003, Consumption-savings decisions with quasi-geometric discounting, Manuscript, Econometrica 71, 365-375. 
[5] Krusell, P., Kuruş̧̧u, B. and A. Smith, 2002, Equilibrium welfare and government policy with quasi-geometric discounting, Journal of Economic Theory 105, 42-72.

[6] Laibson, D., 1997, Golden eggs and hyperbolic discounting, Quarterly Journal of Economics 112, 443-477.

[7] Luttmer, E. and T. Mariotti, 2003, Subjective discounting in an exchange economy, Journal of Political Economy (forthcoming).

\section{Appendix}

Proof to Proposition 1. Guess that the value function $V$ has the following functional form

$$
V\left(a^{\prime}, s^{\prime}\right)=\mu_{0} \exp \left(\mu_{1} a^{\prime}+\mu_{2} s^{\prime}+\mu_{3}\right)
$$

where $\mu_{0}, \mu_{1}, \mu_{2}, \mu_{3}$ are some constant coefficients. Substitution (5) and (11) into equation (3) and the updated version of (4) yields

$$
W(a, s)=\max _{a^{\prime}}\left\{-\frac{1}{\theta} \exp \left[-\theta\left((1+r) a+w s-a^{\prime}\right)\right]+\beta \delta E\left[\mu_{0} \exp \left(\mu_{1} a^{\prime}+\mu_{2} s^{\prime}+\mu_{3}\right)\right]\right\},
$$


and

$V\left(a^{\prime}, s^{\prime}\right)=-\frac{1}{\theta} \exp \left[-\theta\left((1+r) a^{\prime}+w s^{\prime}-a^{\prime \prime}\right)\right]+\delta E\left[\mu_{0} \exp \left(\mu_{1} a^{\prime \prime}+\mu_{2} s^{\prime \prime}+\mu_{3}\right)\right]$

In order to compute the expectations in (12) and (13), we take advantage of the fact that under the assumption of labor productivity shocks (7), $E\left[\exp \left(-\phi \varepsilon^{\prime}\right)\right]$ can be computed analytically:

$$
E\left[\exp \left(-\phi \varepsilon^{\prime}\right)\right]=\int \exp \left(-\phi \varepsilon^{\prime}\right) \cdot \frac{1}{\sigma^{2} \sqrt{2 \pi}} \exp \left(-\frac{\left(\varepsilon^{\prime}\right)^{2}}{2 \sigma^{2}}\right) d \varepsilon^{\prime}=\exp \left(\frac{\phi^{2} \sigma^{2}}{2}\right)
$$

where $\phi$ is a constant.

The first-order condition of (12) with respect to $a^{\prime}$ is

$$
a^{\prime}=\frac{\theta(1+r)}{\theta-\mu_{1}} \cdot a+\frac{\theta w+\mu_{2} \rho}{\theta-\mu_{1}} \cdot s+\frac{1}{\theta-\mu_{1}} \cdot\left(\ln \left(\beta \delta \mu_{0} \mu_{1}\right)+\mu_{3}+\frac{\mu_{2}^{2} \sigma^{2}}{2}\right) .
$$

After updating (14) and substituting it in (13), we obtain

$$
\begin{gathered}
V\left(a^{\prime}, s^{\prime}\right)=\left(-\frac{1}{\theta}+\frac{1}{\beta \mu_{1}}\right) . \\
\exp \left\{\frac{\mu_{1} \theta(1+r) a^{\prime}}{\theta-\mu_{1}}+\frac{\left(\mu_{1} w+\mu_{2} \rho\right) \theta s^{\prime}}{\theta-\mu_{1}}+\frac{\theta\left(\ln \left(\beta \delta \mu_{0} \mu_{1}\right)+\mu_{3}+\frac{\mu_{2}^{2} \sigma^{2}}{2}\right)}{\theta-\mu_{1}}\right\} .
\end{gathered}
$$

The coefficients $\mu_{0}, \mu_{1}, \mu_{2}, \mu_{3}$ are to be such that the functional form of the above function is the same as (11):

$$
\mu_{0}=-\frac{1}{\theta}+\frac{1}{\beta \mu_{1}}, \quad \mu_{1}=\frac{\mu_{1} \theta(1+r)}{\theta-\mu_{1}}, \quad \mu_{2}=\frac{\mu_{1} \theta w+\mu_{2} \theta \rho}{\theta-\mu_{1}}
$$




$$
\mu_{3}=\frac{\theta}{\theta-\mu_{1}} \cdot\left(\ln \left(\beta \delta \mu_{0} \mu_{1}\right)+\mu_{3}+\frac{\mu_{2}^{2} \sigma^{2}}{2}\right)
$$

Solving the system of four equations with respect to four unknowns $\mu_{0}, \mu_{1}$, $\mu_{2}, \mu_{3}$ and substituting the solution into (11), we obtain the formula for the optimal value function $V$ in the main text. Finally, by substituting $\mu_{0}$, $\mu_{1}, \mu_{2}, \mu_{3}$ and $a^{\prime}$ given by formula (14) into (12), we get the optimal value function $W . \quad \|$ 